\title{
Very low-carbohydrate high-fat diets are superior to low-fat diets in improving cardiovascular markers: meta-analysis of large, long-term randomised controlled trials
}

\author{
Teuta Gjuladin-Hellon ${ }^{1,2}$, Ian Davies ${ }^{2}$, Jackie Fealey ${ }^{2}$, Alexander Montasem ${ }^{3}$ and Katie Lane ${ }^{2}$ \\ ${ }^{1}$ University of Central Lancashire, School of Medicine, Preston, United Kingdom, \\ ${ }^{2}$ Liverpool John Moores University, School of Sport Studies, Leisure and Nutrition, Liverpool, United Kingdom and \\ ${ }^{3}$ University of Central Lancashire, School of Medicine, Preston, United Kingdom
}

\section{Abstract}

Our recent study (1) showed that the amount of dietary carbohydrates in obesity interventions has differential effects on cardiovascular risk markers (CVM) and effects magnitude depends on intervention duration. Very-low carbohydrate high-fat diets (VLCD) were superior in ameliorating lipid markers compared to high-carbohydrate low-fat diets (LFD).

We updated our systematic review and meta-analysis to include long-term effects of VLCD ( $<50 \mathrm{~g} /$ day) on weight, glucose, total cholesterol, insulin and blood pressure (BP) among overweight/obese adults in comparison to LFD.

Medline, PubMed, Cochrane Central, and CINAHLPlus were searched to identify large ( $>100)$ randomised controlled trials (RCT) with duration $\geq 6$ months. Risk of bias, a random effects model and subgroup analyses based on duration of follow-up were performed using Review Manager. Results were reported according to PRISMA.

Four open label RCT ( $=723 ; 362$ VLCD; 361 LFD) with some form of behavioral intervention and duration 6-24 months were identified. VLCD showed more favorable effects on diastolic BP at 6 months $(-1.96 ; 95 \% \mathrm{CI},-2.99$ to $00.93 ; \mathrm{P}=0.0002)$ and 24 months $(-2.69 ; 95 \% \mathrm{CI},-4.87$ to $-0.51 ; \mathrm{P}=0.001)$, near significant level at 12 months $(-1.79 ; 95 \% \mathrm{CI},-3.56$ to $0.04 ; \mathrm{P}=0.05)$ and an overall total favourable effect $(-1.98 ; 95 \% \mathrm{CI},-2.73$ to -1.22$)$. The decrease in systolic BP was greater among VLCD for the whole period and the overall total effect reached the level of significance $(-1.76 ; 95 \% \mathrm{CI},-3.56$ to $0.04 ; \mathrm{P}=0.05)$. VLCD showed beneficial effect on total cholesterol level at 6 and 12 months $(-0.01 \mathrm{mmol} / \mathrm{L} ; 95 \% \mathrm{CI},-0.01$ to $-0.00 ; \mathrm{P}=0.002$ and $-0.01 \mathrm{mmol} / \mathrm{L}$; $95 \% \mathrm{CI},-0.01$ to $-0.00 ; \mathrm{P}=0.005$, respectively). The mean changes in weight, and fasting glucose and insulin levels revealed non-significant differences between both diets at any measured time, although these parameters decreased within both groups compared to baseline.

VLCD led to significant total weighted mean decrease of diastolic BP and near significant decrease of systolic BP independent of changes in body weight, fasting glucose or insulin levels. The present data on decreased levels of diastolic BP and total cholesterol, combined with our recently published results on increased HDL-cholesterol, decreased triglycerides and no significant effect on LDL-cholesterol (1) provide evidence that VLCD are superior to LFD in improving traditional CVM in longer term.

\section{Conflict of Interest}

Authors declare no conflict of interest

\section{Reference}

1. Gjuladin-Hellon et al. (2019) Effects of carbohydrate-restricted diets on low-density lipoprotein cholesterol levels in overweight and obese adults: a systematic review and meta-analysis. Nutrition Reviews 77(3), 161-180. doi.org/10.1093/nutrit/nuy049 